\title{
Virtual Reality as a Platform for Research in Gambling Behaviour
}

\author{
Patrick Dickinson ${ }^{1}$, Kathrin Gerling ${ }^{2}$, Liam Wilson ${ }^{1}$, Adrian Parke ${ }^{3}$
}

\begin{abstract}
Research into gambling behaviour is an important aspect of mitigating the effects of problem gambling. However, the majority of existing studies are conducted in the laboratory, rather than in vivo, raising questions over the generalisability of results. Virtual reality (VR) is well established as an effective tool for exposure therapy, and has also been used in other experimental contexts: such work is often motivated by the ability of the platform to create ecologically valid conditions, and simulations that would be hard to create in real life. Whilst a small number of studies have used VR to evoke the urge to gamble, and also to conduct behavioural studies, no work has yet considered the differences in experience between laboratory and VR conditions.

We approach this from the perspective of immersion, arousal and user experience, and conduct a within-subjects study $(\mathrm{N}=48)$ in which participants play a gambling game both on a laptop in a laboratory, and on a virtual gaming machine located in a VR simulation of a betting shop. Our results show that participants reported higher levels of arousal, as well as higher levels of immersion in the game when playing in VR. There was also a significant difference in self-reported physical task workload in VR. Based on our results, we argue for further use of VR in experimental research related to gambling, as well as further study of the experience and responses of problem gamblers to VR simulations of real-world gambling.
\end{abstract}

Keywords: virtual reality, gambling behaviour, user experience

\section{Introduction}

Gambling represents a significant sub-sector of the UK leisure industry. The gambling commission estimates that $63 \%$ of adults engage in some kind of gambling activity each year. However, a significant number of gamblers suffer the effects of addiction and associated negative outcomes: in 2017, the commission estimated that there were approximately 300,000 "problem gamblers" in the UK, with at least 500,000 more at moderate risk [1, 2].

A significant body of research has been conducted into the psychology of gambling, with the objective of understanding and mitigating factors which lead to problem behaviour. This includes, for example, player responses to game features, environmental factors, and the effectiveness of interventions (e.g. [3, 4, 5, 6, 7]). However, the majority of experimental research is conducted in laboratory settings, rather than in vivo, leading to questions over the generalisability of results $[8,9]$.

In this paper we present an investigation of virtual reality (VR) as a platform to support research into gambling behaviour. Work in other contexts has shown that VR systems can combine ecological validity with high levels of control, and this has frequently been leveraged for psychological treatments such as exposure therapy [10, 11, 12, 13].

\footnotetext{
${ }^{1}$ School of Computer Science, University of Lincoln, UK

2 eMedia Research Lab, KU Leuven, Leuven, Belgium

${ }^{3}$ School of Media, Culture and Society, University of the West of Scotland, UK
}

A small number of studies have investigated VR as a therapeutic tool for problem gambling [14, 15. However, the use of VR as a platform for behavioural research in gambling warrants a closer consideration of user experience and response: as with laboratory settings, researchers need to be able to make informed judgements about participant engagement, the generalisability of results, and the design of simulations and experimental scenarios. No existing work has yet addressed this.

Our motivation is thus to explore whether VR might be used to provide more effective experimental environments for gambling research than the laboratory. Gambling research presents particular challenges in terms of ecological validity, so we approach this from a user experience perspective. We present results from a within subjects study $(\mathrm{N}=48)$ in which we evaluate immersion, affective response and usability for a touch-screen gambling game, played both in a laboratory environment, and on a simulated electronic gaming machine (EGM) in a VR betting shop. Our results show self-reported increases in player immersion in the gambling game while in the VR condition, along with increased feelings of arousal. Our results also show an associated increase in self reported physical task workload when using VR. No previous work has undertaken a comparable analysis.

Based on our results, we argue for the wider use of VR in gambling research, and also propose that further work with sub groups of regular and problem gamblers is warranted. The remainder of our paper is structured as fol- 
lows. In Section 2 we present the background to our work. This comprises a discussion of user experience in VR systems, existing use of VR in psychology research, including applications to gambling exposure. We then proceed to discuss our motivation and experimental hypotheses, and methodology. We conclude by presenting the results or our study, with discussions and conclusions.

\section{Background}

In this section we first briefly review concepts of presence and immersion in VR and games. We then proceed to discuss how VR has been leveraged by psychology researchers both in therapeutic and experimental contexts, and the relatively small amount of existing work which has used VR in gambling-related settings. This is followed by an overview of our study.

\subsection{Presence and Immersion}

VR simulations are characterised by a strong sense of presence - a feeling of "being there", or of being present in another physical space [16]. This phenomenon has been extensively investigated and characterised: for example, Witmer and Singer [17] define presence as "the subjective experience of being in one place or environment, even when one is physically situated in another", and Slater and Wilbur [18] describe it as "a state of consciousness, the (psychological) sense of being in the virtual environment.". Some authors have made attempts to further deconstruct presence. Slater [19] defines the distinct terms place illusion, which refers to the said sense of "being there", and plausibility illusion to the sense that what is happening in the simulation is real. Slater further discriminates between these by attributing them to perceptual and cognitive process respectively. A recent review is presented by Skarbez et al. [20, which proposes a synthesised model of presence comprising elements of place illusion, plausibility illusion, and social presence, and summarises commonly used selfreport measures (e.g. [17, 21]).

In contrast to presence, the term immersion refers to the sense of involvement or absorption in an activity, and is often used to characterise users' engagement with games and other technologically mediated experiences (including VR). However, presence and immersion are distinct and different concepts. Slater 22] describes the difference as that of form (presence) and content (immersion). Brown and Cairns 23. also discuss the relationship between immersion and presence, within the context of games, and conclude that (for game players) immersion encompasses a spectrum of meanings, which may converge with feelings of presence when experienced in high-intensity. Jennet et al. 24 have also described immersion in games (e.g. playing a video game on a 2D screen) as a user's sense of engagement and involvement in game play, and proposed five factors that capture this experience: cognitive involvement, real-world disassociation, emotional involvement, challenge, and control, which may be evaluated with the widely used Immersive Experiences Questionnaire (IEQ). This measure captures a number of established and studied components which together create the sense of involvement and absorption experienced by players, including emotional and cognitive involvement in the game, and presence 23. The concept of immersion also overlaps with that of game flow [25, 26]. The scales have been validated by Jennett et al. and also associated with objective measures such as task completion times, and eye movements. We return to the IEQ when discussing the evaluation of player experience in our own study, in Section 4.

There is also further confusion over the terms presence and immersion specifically in relation to VR. In particular, Slater uses the term immersion to refer to the technical characteristics of a VR system (e.g. the resolution of a head mounted display, or fidelity of a tracking system [27]), and this terminology is widely adopted by VR researchers. For clarity, we henceforth use the term system immersivity to refer to Slater's definition based on technical characteristics of VR, and immersion to refer to the multi-dimensional sense of engagement and involvement described by Brown and Cairns, and Jennett et al.

\subsubsection{Presence and Affective Response}

Affect is an important factor in understanding gambling behaviour (e.g. [28, 29, 30]), and it is therefore useful to consider how VR mediates affective responses. Work by Riva et al. 31 investigated the ability of VR scenes to invoke specific emotions (anxiety and relaxation), and reported an inter-relationship between presence and emotional response. A number of studies have noted increased arousal when performing tasks in VR (HMD), as compared to $2 \mathrm{D}$ screen-based interfaces. For example Estupiñán et al. 32, and Kim et al. 33] who found higher arousal, irrespective of task stress. A recent review of the relationship between presence and emotion is presented by Diemar et al. 34], from the perspective of exposure therapy. They surmise that system immersivity can amplify arousal, resulting in reported elevation of emotional responses(e.g. see Visch et al. [35]).

Of particular relevance to our study is work by Villani et al. 36] which compared self-reported presence during a job interview simulated in the real-world, with a VR simulation of the same interview. Users reported elevated presence and anxiety in the VR condition, which was attributed to the use of a more contextually correct environment (boardroom), as opposed to the use of a lecture room for the real-world interviews. This comparison of real and VR experiences mirrors our own experimental design, and partially motivates our study.

\subsection{VR and Psychology Research}

VR technologies have been explored extensively for therapeutic uses, such as exposure therapy (VRET), which leverages users' sense of presence to create experiences which can replace in vivo methods. Recently, GarciaPalacios et. al. 37] showed that VR was effective in 
treating arachnophobia, noting the advantages of environmental control. Other examples include treatment of acrophobia 38], fear of flying [39, social anxiety disorder [10], and post traumatic stress disorder 11. A recent metaanalysis of randomized control trials shows that VRET, examined across a range of conditions, is generally as effective as in vivo therapy [40]. The concept of ecological validity frequently appears in discussions of VRET; however, there is relatively little consideration of how the design of experiences moderates successful treatment, beyond observations that VR facilitates levels of control and personalisation which are not possible in vivo.

\subsubsection{VR as a Platform for Experimental Research}

A smaller body of work has also considered VR as a tool for experimental psychology, and is similarly built on propositions of ecology validity, experimental control, and the ability to construct contexts which would be hard to reproduce in real life. However, the question of how well results might generalise has been highlighted as an open challenge by a number of researchers, and motivates our own work.

Gaggioli 41] presented a discussion of applications to experimental themes in cognitive psychology, such as perception, attention, and cognitive performance. Wilson and Soranzo 42 discussed the application of VR to research in visual perception, while Bohill et al. 43. reviewed the use of VR in experimental neuroscience. VR has also attracted the attention of social psychologists. Recently, Pan and Hamilton 44 discussed the use of VR to conduct experimental work in human behavioural interactions, noting the importance of plausibility illusion ([19]) in maintaining a sense of presence. Kulik [45], commenting on Pan and Hamilton's paper, further underlined that more investigation is required to validate behavioural research findings obtained in VR simulations.

\subsubsection{VR and Gambling Research}

A small amount of existing research has explored the use of VR in settings related to gambling. This is mainly related to exposure therapy, and representation of settings intended to evoke the urge to gamble. For example, Giroux et al. 46] investigated the use of a VR simulation to induce the desire to play Video Lottery Terminal (VLT) machines among regular players, and found that the simulation elevated participants' desire to play. Similarly, Park et al. 14 used a virtual environment to measure the effect of repeated exposure on recreational users' urge to gamble. They reported that initial exposure created an elevated urge, which decreased over time. Similar work by Loranger et al. 47] showed that VR simulations of a bar and casino could be used to invoke the desire to gamble, and subsequent work by Bouchard et al. 15, showed that these stimuli can be effectively integrated into Cognitive Behavioural Therapy (CBT) treatments for gambling disorder.
Of most relevance to our own work, Young et al. 48 used a semi-immersive VR platform (HMD in combination with joystick and mouse controls) to study persistence of slot machine play. Further work using the same VR system has been undertaken by Wohl et al. [49, Lister et al. [50, and Hollingshead et al. [51] to investigate chasing behaviour, and players' response to in-game pop-ups respectively. While these studies provide interesting results regarding specific behaviours, there is a lack of existing work which explores the general experience and response of participants whilst in VR gambling simulations, particularly compared to other experimental environments: this is the focus of our work, which provides context and support for the validity of these (and other future) studies.

\subsection{User Experience and Gambling}

We take a user experience perspective, and so briefly summarise research related to the experience of gamblers relevant to our study constructs. The experience of gambling is generally characterised as "excitement", or an elevated sense of arousal during play. Arousal is a complex physiological response; however, we refer to the recent discussion by Pijeira-Daz et. al. 52, which describes a "physiological activation and responsiveness triggered by an event, object, or situation, during a person's interaction with the environment." Arousal associated with the excitment of gambling has been evaluated using physiological measures such as heart rate [8, 53], subjective self-report measures (e.g. 30]), and sometimes both. Significant correlations between physiological and self-report measures of arousal have been found [29, 54, although this is not universally reported (e.g. 55]). Nevertheless, some relationship between what may be considered as objective (physiological) measures, and subjective measures of affective state seems apparent, if not well understood, in previous work.

The experiences of gamblers during play has also been characterised as a sense of absorption or focus, closely related to that described in Section 2.1 for video game players [24]. For example, Schull [56] described a "dissociated subjective state that gamblers call the zone, in which conventional spatial, bodily, monetary, and temporal parameters are suspended". Dixon et al. 57 recently described slot machine play as "dark flow", drawing parallels with the flow state experienced in video games [25, 26], and Murch and Clarke [58] also recently used video game research constructs to measure immersive flow experiences in slot machine players.

\section{Study Overview}

Studies in gambling behaviour typically make use of laboratory-based experiments, which provides high experimental control, at the cost of reduced generalisability (e.g. [9]). However, the literature indicates that VR can offer 
both control and good ecological validity. We are therefore motivated to ask whether it can provide a more effective platform for gambling behaviour research than laboratory settings, how participant experiences might differ, and whether those experiences might better generalise. Although researchers are starting to explore VR as tool for gambling research (e.g. [48, 49, 50]), these questions have not yet received consideration.

\subsection{Aspects of User Experience}

A comparison of laboratory and in vivo study settings for gambling research has been presented by Gainsbury and Blaszczynski [9], which discusses a number of relevant aspects. In particular, arousal has been strongly linked with persistent gambling: Anderson and Brown [8] found lower levels of arousal in laboratory settings; Diskin et al. 55] also found that arousal among EGM players was more elevated in a real-world setting than in the laboratory. However, such comparisons are sparse. Furthermore, We also wish to consider players' sense of immersion (in the sense of absorption and involvement) in play, as this has been identified as a characteristic of gambling (particularly slot machine play [57) in Section 2.3.

In addition, we wish to determine whether there are any extra physical, cognitive or mental demands required to undertake gambling tasks using a VR system, as this could also affect player behaviour and consequently affect the generalisability of results. Users' perceptions of required effort are referred to as task workload, which is described by Hart [59] as " a term that represents the cost of accomplishing mission requirements for the human operator". Workload is typically characterised by a subjective rating of users' perceived effort on a number of dimensions: mental, physical, and temporal demands, user frustration, and perceived effort and performance. In the context of our study, increased workload could potentially arise from additional physical or cognitive effort required to operate the VR system, or limitations of system immersivity, for example.

\subsection{Research Questions}

We compare the experience of participants playing an electronic gambling game on a real touchscreen in a laboratory setting, to that of playing the same game on a virtual touchscreen machine, in a VR representation of a UK betting shop. In particular, we ask the following research questions (which we develop into specific hypotheses in Section 4.2):

RQ1: Do users experience higher levels of immersion (engagement and involvement) in the gambling game, while playing it in the VR setting, as compared to the laboratory?

RQ2: Do users experience higher levels of arousal while playing the game in the VR environment, as compared to the laboratory setting?
RQ3: Is there any difference in perceived task workload for players while playing the touch-screen gambling game in VR, as compared to the laboratory?

\subsection{Gambling Component: Five Card Draw}

We created a simulated gambling video game called "Five Card Draw" (FCD) using the Unity3D game engine. The game is representative of real EGM games, comprising typical game-play elements. Players have an in-game balance (initially $£ 50$ ), and each play costs $£ 1$. For each play, five cards are initially drawn, and the player then chooses to hold between one and five of those cards. Non-held cards are discarded and replaced from the pack to create the final hand, which may be either winning or losing. Winning hands are based on typical poker hands: pairs of face cards, two pairs, three of a kind, full house, flush, straight, four of a kind and straight flush. Each winning hand has a different value, and in-game currency is fictitious: players do not play with real money. If the player draws a winning hand, they may additionally choose to gamble those winnings on a separate high-low card draw, where they can double (or lose) their winnings from that hand. Each hand takes a few seconds to play.

The FCD game can be played either on a touch-screen laptop or tablet, or on a virtual EGM, in a VR betting shop simulation. The same game code is used in each case, and the game plays identically. The laptop version is played by touching flashing buttons on the screen, which the player uses to start the game, hold cards, and make choices in the high-low game phase. The VR version is played using the HTC Vive VR platform, comprising a head mounted display (HMD) and hand-held game controllers. The Vive controllers appear in the simulation as hands, which the player can use to virtually touch the same screen controls on the simulated EGM machine. Figure 1 shows FCD running on a laptop. Figure 2 shows part of the simulated VR betting shop, and Figure 3 shows an array of simulated EGMs in the betting shop: FCD is running on the EGM closest to the view point in this image. Figure 4 shows an image taken while playing the FCD game in VR.

The VR simulation was designed to incorporate typical elements of a UK betting shop environment, including items such as paper slips, pens, stools and tables. There were also large screens showing video excerpts from discussions by racing analysts, with audio. These are also typical of UK betting shops, and provided a naturalistic audio soundtrack to isolate the player from the real world. While in the VR simulation, the participant may move around by walking, which restricts movement to be within a few feet of the EGM that they are playing. They were therefore unable to interact with other parts of the simulated environment. No simulated characters were placed in the environment, so as not to unduly distract participants while playing the EGM.

The outcome of each hand of FCD is partially dependent on choices made by the player (e.g. which cards to hold, whether to play high-low); however, outcomes also 


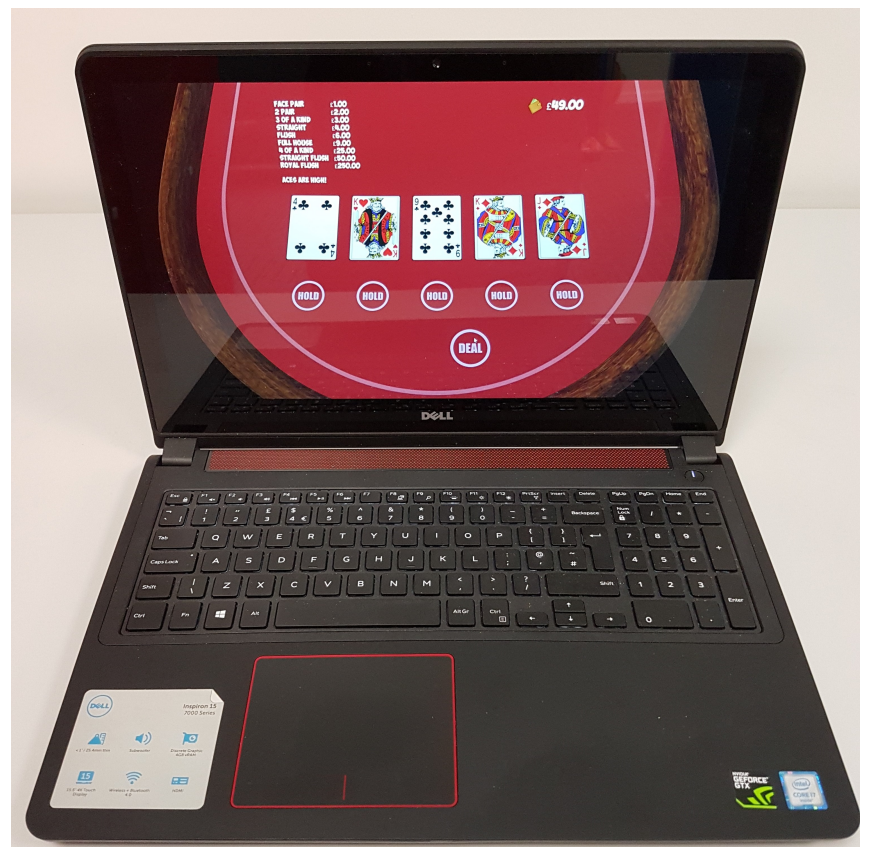

Figure 1: FCD on a Touchscreen Laptop

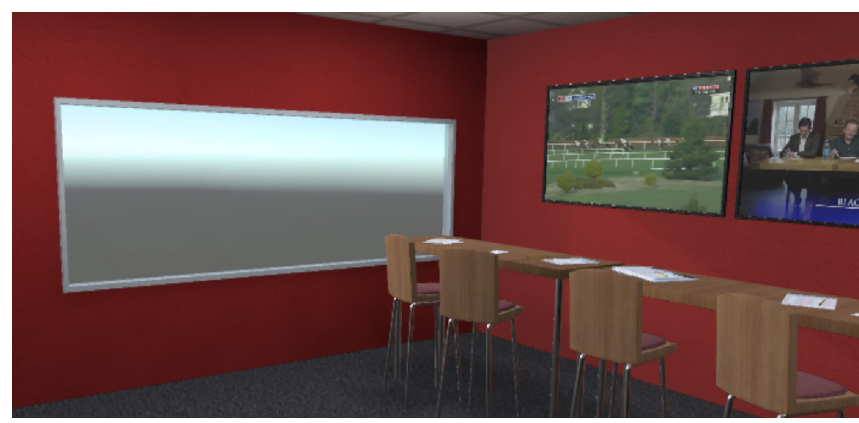

Figure 2: The simulated betting shop

depend on the game's response to those choices. The FCD game was therefore programmed so that the game's responses can be controlled by the experimenter, in order to create comparable game outcomes for participants. This was effected by defining a target balance for each hand in a sequence of play: the game selects initial cards, replacement cards, and cards for the high-low game in order to keep the player's total balance close to the target. For example, if the target is less than the player's current balance, the game will deal a losing hand. If it is greater, then it will deal a winning hand with a win value equal to or less than that required to match the target value: the high-low game is then chosen to either win or lose depending on which outcome takes the player's total balance closest to the target. Within these parameters, cards are randomised, including initially dealt hands.

\section{Experimental Method}

In order to address the research questions defined in Section 3 we designed a two-condition, within-subjects

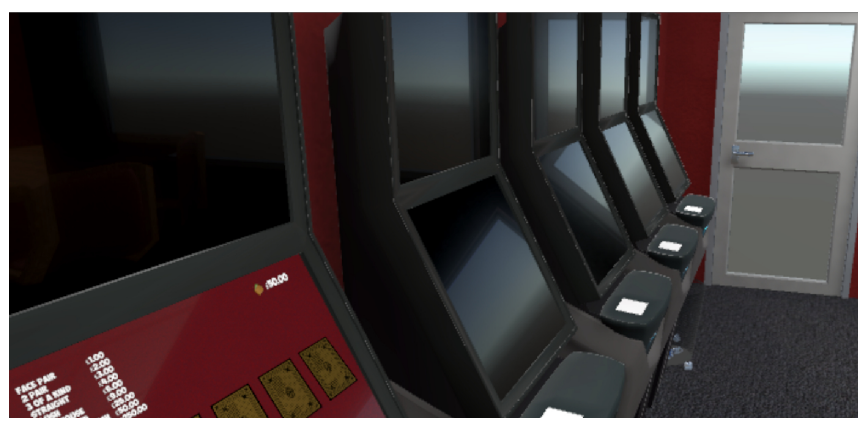

Figure 3: simulated EGMs in VR

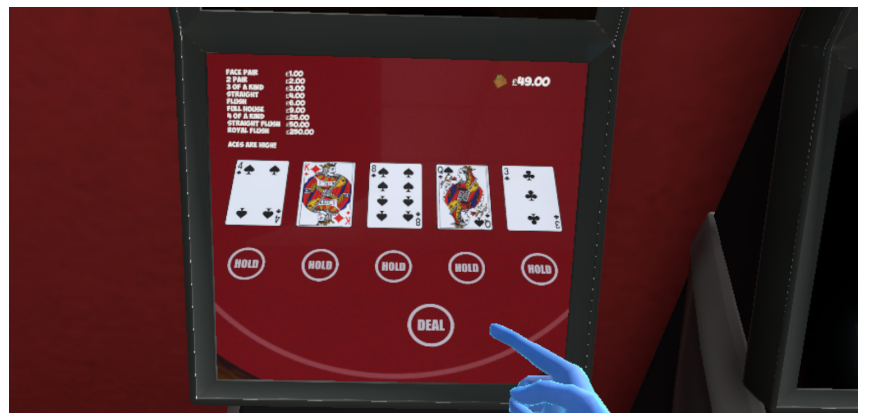

Figure 4: Playing FCD on the simulated EGM in VR

study to compare participants' experience of playing FCD in the laboratory on a touch screen laptop, with playing it on the virtual EGM, within the VR simulation. Our study was approved by the Ethics Committee of the University of Lincoln prior to being conducted.

\subsection{Participants}

We recruited 48 participants (32 male, 16 female) for our study, from the local population. Ages ranged from 18 to $51(\mathrm{M}=25.66, \mathrm{SD}=8.17)$. Participants were recruited by word of mouth, required to be at least 18 years old, and predominantly comprised students and staff from the University of Lincoln. Thirty nine had previously used VR equipment on at least one occasion, and twenty five reported that they had played a gambling machine in the last year.

Prior to undertaking the study, participants were screened using a questionnaire to identify any conditions which might exclude them from using VR safely, such as vulnerability to falls, or restricted mobility, and also for uncorrected visual impairments. We also screened participants to exclude those with current or previous problems related to gambling behaviour.

\subsection{Measures}

We employed a number of self-report measures, which were completed by participants after each condition, and which correspond to the research questions defined in Section 3 . 
- We used the Immersive Experiences Questionnaire (IEQ) 24 to assess participants' sense of immersion (engagement and involvment) in the FCD game, and address RQ1. We chose this scale as it has been validated and widely used in video games research, and overlaps closely with concepts of immersion described for players of electronic gambling machines in Section 2.3. The IEQ comprises 31 items, each reported on a seven point Likert scale (e.g. "To what extent did you feel you were focused on the game?"). These are scored as 5 sub-scales: challenge, control, real-world disassociation, emotional involvement and cognitive involvement. The IEQ demonstrated good scale internal reliability, with Cronbach's alpha scores of 0.83 and 0.82 in the laptop and VR conditions respectively.

- RQ2 refers to users' sense of arousal during play. Some previous works have used physiological measures, such as skin conductance, to measure arousal (e.g. 55]). However, such measures are prone to noise caused by physical movement, and are thus less suitable for VR conditions. We therefore used the Self-Assessment Manikin (SAM) 60, as an alternative self-report measure of affective state, which includes arousal as a dimension. The SAM comprises pictorial representations of valence, arousal, and dominance, and each is reported on a nine-point scale.

- We used the NASA TLX questionnaire 59 to assess perceived task workload, and address RQ3. This comprises six items: physical demand, mental demand, temporal demand, performance, frustration, and effort (e.g. "Physical Demand: How physically demanding was the task?"). Each is reported on a 21-point scale.

\subsection{Hypotheses}

We constructed the following hypotheses, with respect to the chosen measures and RQ1-RQ3:

H1: Participants' reported level of immersion in the FCD game while playing in the VR environment will be higher than that experienced while playing on the laptop. We further propose the auxiliary hypothesis that this will be evident across all sub-scales apart from challenge (as the game mechanics are identical). This hypothesis is motivated by discussions in the literature regarding the high ecological validity of VR (e.g. [41, 44, 61]): we conjecture that participants' sense of being present in a betting shop will result in a higher level of immersion/engagement in the FCD game.

H2: Participants' level of self-reported arousal will be higher while playing FCD in the VR environment than while playing on the laptop. This is partly motivated by Villani et al. [36] who have shown that higher levels of presence and emotional response may be seen in VR compared to real world settings, when the VR environment provides more meaningful context. It is further motivated by previous findings that higher levels of arousal may be seen in vivo (e.g. [8, 55]), compared to non-naturalistic settings. We conjecture that the comparatively higher ecological validity of the VR environment will evoke higher self-reported levels of arousal, as discussed in Section 2.3.

H3: Participants will experience higher level of task workloading while playing the VR version of the game. This is motivated by the observation that using the HMD and hand controllers of the HTC Vive may require some physical or cognitive effort, and also that the VR touchscreen, which has no physical manifestation, may induce further motor-sensory load.

\subsection{Procedure}

Participants were given an information sheet about the study, and then provided informed consent, in line with ethical requirements of the University of Lincoln. A brief demographic questionnaire was also completed by participants, which provided information about age and gender, whether they had previously used VR equipment, and whether they had recently used a gambling machine.

Participants were then introduced to the FCD game. This was done using a Powerpoint presentation which described the rules of the game, winning hands, game phases, and showed screen shots from the game to explain the screen layout. Participants controlled the presentation themselves, and could move forwards and backwards through the slides until they were happy that they understood the game. Platform-specific elements were not shown in the presentation, so that participants' understanding of the FCD game mechanics and outcomes was independent of either the laptop and VR platforms on which it was to be played.

Participants were then told that they would be playing the FCD game twice, once on a laptop, and once on a simulated gaming machine in a VR environment, and that after each condition they would be asked to fill in three questionnaires relating to their experience of playing the game. We additionally explained the SAM questionnaire prior to starting the conditions. Participants were also told that their final in-game currency balances at the end of each condition would be converted into lottery tokens, and that those tokens would be entered into a randomised draw for two $£ 50$ gift vouchers, at the end of the study. This was intended to incentivise players to win as much in-game currency as possible.

Participants then started the conditions. Half played the VR condition first, and the other half played the laptop condition first: participants were alternately assigned. Prior to each condition, participants were instructed on how to play the game on the respective platform. For the VR condition, this first included a short training session in an empty virtual environment, which showed participants how to use the headset, controllers, and safety features; and how to control the hands inside the simulation. 
In each condition, participants played 35 hands of FCD. We used the same target balance sequence for each condition, for each participant. The sequence had some small arbitrary peaks and troughs but the final target balance was the same as the initial balance. This created a game experience which contained a series of moderate wins and losses, but an overall neutral gambling experience: that is, all players finished with an in-game currency balance close to their initial balance of $£ 50$. After each condition, players completed the SAM, NASA-TLX, and IEQ questionnaires. On completion of each condition, participants were given a record of their tokens for the randomised giftcard draw.

\section{Results}

We present results for each of the self-report measures, for each condition. In each case, we used a mixed ANOVA to determine significance, at the $\alpha=0.05$ level, and relate results back to our hypotheses $\mathrm{H} 1$ to $\mathrm{H} 3$. We used mixed ANOVAs rather than pairwise T-tests because, although we alternated the order of conditions, we wished to rule out any other possible contrast effects cause by condition order. We therefore used platform type (laptop or VR) as the within-subjects factor, condition order as the betweensubject factor, and report full results for all measures in Table 1

In the remainder of this section we address each measure in turn, and summarise our results, focussing on the within-subject factor which describes differences between participants' experiences of playing the laptop and VR conditions. Where appropriate, we remark on any other salient features.

\subsection{Immersion}

Total reported immersion using the IEQ was higher for the VR version of the FCD game $(\mathrm{M}=155.08$, SD $=22.89)$ than for the laptop version $(\mathrm{M}=135.67, \mathrm{SD}=$ 24.37). The main effect of condition type (VR or laptop), was statistically significant $\mathrm{F}(1,46)=32.58, \mathrm{p}<0.001, \eta_{p}^{2}$ $=0.42$. Furthermore, there was no statistically significant interaction when including order effect as a fixed factor $\mathrm{F}(1,46)=1.62, \mathrm{p}=0.209$. We next consider each subscale individually, in turn.

There was no significant main effect of challenge $\mathrm{F}(1$, $46)=0.28, \mathrm{p}=0.597$, and no interaction was observed after introducing the order of condition presentation as a fixed factor $\mathrm{F}(1,46)=0.14, \mathrm{p}=0.714$. However, participants did report higher feelings of control in the VR condition $(\mathrm{M}=26.63, \mathrm{SD}=4.36)$ than in the Laptop condition $(\mathrm{M}=22.63, \mathrm{SD}=5.42)$, and this difference was statistically significant $\mathrm{F}(1,46)=22.78, \mathrm{p}<0.001, \eta_{p}^{2}=$ 0.33 . There was no statistically significant interaction between feelings of control and the order of the conditions $\mathrm{F}(1,46)=0.99, \mathrm{p}=0.325$. Experience of real world dissociation was also substantially higher when using VR (M
$=35.77, \mathrm{SD}=7.14)$, compared to using the laptop $(\mathrm{M}=$ $25.58, \mathrm{SD}=8.14$ ), and this main effect of condition type was statistically significant $\mathrm{F}(1,46)=69.09, \mathrm{p}<0.001$, $\eta_{p}^{2}=0.6$. However, there was a statistically significant interaction between condition type and the condition order $\mathrm{F}(1,46)=7.31, \mathrm{p}=0.01, \eta_{p}^{2}=0.14$. Participants experienced higher levels of real world dissociation when playing FCD on the laptop before playing it on VR $(\mathrm{M}=28.67$, $\mathrm{SD}=9.54)$ compared to playing it after the VR condition $(\mathrm{M}=22.5, \mathrm{SD}=4.94)$.

Participants further reported higher levels of emotional involvement within the $\mathrm{VR}$ condition $(\mathrm{M}=26.96, \mathrm{SD}=$ $6.59)$ than in the laptop condition $(\mathrm{M}=24.19, \mathrm{SD}=6.45)$, and this main effect was statistically significant: $\mathrm{F}(1,46)$ $=9.71, \mathrm{p}=0.003, \eta_{p}^{2}=0.17$. There was no significant interaction between level of emotional involvement in each condition and the order in which the conditions were presented: $\mathrm{F}(1,46)=0.014, \mathrm{p}=0.907$. Participants also experienced higher levels of cognitive involvement in VR $(\mathrm{M}=47.81, \mathrm{SD}=7.22)$ than when playing the laptop condition $(\mathrm{M}=45.63, \mathrm{SD}=7.15)$, and this main effect was statistically significant $\mathrm{F}(1,46)=5.15, \mathrm{p}=0.028, \eta_{p}^{2}=$ 0.10 . There was no significant interaction between factors $\mathrm{F}(1,46)=0.079, \mathrm{p}=0.78$.

Based on these results, our hypothesis H1, that participants would experience higher levels of immersion playing the FCD game in the VR condition, is supported. Moreover, with the exception of real world dissociation, there was no statistically significant interaction with condition order. The difference in levels between VR and laptop gambling were most pronounced with respect to the real world dissociation sub-scale. Therefore, it is unsurprising to observe an interaction in this case: the effect was so large in VR that being presented with the VR condition first appeared to reduce the effect when subsequently playing on the laptop. In addition, the auxiliary hypothesis is also supported: participants did not report higher levels of challenge using VR.

\subsection{Affective Response}

Participants used the Self-Assessment Manikin to report affective response to the conditions on three dimensions: valence, arousal and dominance. They reported higher levels of arousal in response to the VR condition $(\mathrm{M}=5.69, \mathrm{SD}=1.64)$, in contrast to the laptop condition $(\mathrm{M}=5.13, \mathrm{SD}=1.92)$, and this main effect for condition type was statistically significant: $\mathrm{F}(1,46)=4.78$, p $=0.034, \eta_{p}^{2}=0.094$. There was no significant interaction with condition order: $F(1,46)=2.37, \mathrm{p}=0.131$. Similarly, participants reported higher feelings of dominance in the VR condition $(\mathrm{M}=5.77, \mathrm{SD}=1.36)$ than the laptop condition $(\mathrm{M}=5.33, \mathrm{SD}=1.36)$ : this was statistically significant $\mathrm{F}(1,46)=5.43, \mathrm{p}=0.024, \eta_{p}^{2}=0.11$, despite the differences in means being relatively small. However, a significant interaction with condition order was observed: $\mathrm{F}(1,46)=11.84, \mathrm{p}=0.001, \eta_{p}^{2}=0.21$. The findings show 


\begin{tabular}{|c|c|c|c|c|}
\hline Scale & Within Subjects & Between Subjects & Laptop First & VR First \\
\hline & $\begin{array}{l}\text { (Laptop) } \\
\text { (VR) }\end{array}$ & $\begin{array}{c}\text { (Laptop First) } \\
\text { (VR First) }\end{array}$ & $\begin{array}{l}\text { (Laptop) } \\
\text { (VR) }\end{array}$ & $\begin{array}{l}\text { (Laptop) } \\
\text { (VR) }\end{array}$ \\
\hline SAM & \multicolumn{4}{|l|}{ Valence } \\
\hline $\mathrm{M}(\mathrm{SD})$ & $6.54(1.30)$ & 6.85 & $6.83(1.17)$ & $6.25(1.39)$ \\
\hline $\mathrm{M}(\mathrm{SD})$ & $6.98(1.56)$ & 6.67 & $6.88(1.87)$ & $7.08(1.21)$ \\
\hline \multirow[t]{2}{*}{$\mathrm{F}(1,46), \mathrm{p}, \eta_{p}^{2}$} & $3.57,0.065,0.072$ & $0.30,0.589,0.006$ & \multicolumn{2}{|c|}{ Interaction : $2.93,0.094,0.060$} \\
\hline & \multicolumn{4}{|l|}{ Arousal } \\
\hline $\mathrm{M}(\mathrm{SD})$ & $5.13(1.92)$ & 5.67 & $5.58(1.91)$ & $4.67(1.86)$ \\
\hline $\mathrm{M}(\mathrm{SD})$ & $5.69(1.64)$ & 5.15 & $5.75(1.68)$ & $5.63(1.64)$ \\
\hline \multirow[t]{2}{*}{$\mathrm{F}, \mathrm{p}, \eta_{p}^{2}$} & $4.78,0.034,0.094$ & $1.39,0.245,0.029$ & Interaction : & $37,0.131,0.049$ \\
\hline & \multicolumn{4}{|l|}{ Dominance } \\
\hline $\mathrm{M}(\mathrm{SD})$ & $5.33(1.36)$ & 5.60 & $5.71(1.52)$ & $4.96(1.08)$ \\
\hline $\mathrm{M}(\mathrm{SD})$ & $5.77(1.36)$ & 5.50 & $5.50(1.35)$ & $6.04(1.33)$ \\
\hline $\mathrm{F}, \mathrm{p}, \eta_{p}^{2}$ & $5.43,0.024,0.11$ & $0.097,0.757,0.002$ & Interaction : & $.84,0.001,0.21$ \\
\hline IEQ & \multicolumn{4}{|l|}{ Challenge } \\
\hline $\mathrm{M}(\mathrm{SD})$ & $17.65(3.56)$ & 18.77 & $18.54(3.55)$ & $16.75(3.40)$ \\
\hline $\mathrm{M}(\mathrm{SD})$ & $17.92(3.80)$ & 16.79 & $19.00(3.51)$ & $16.83(3.83)$ \\
\hline \multirow[t]{2}{*}{$\mathrm{F}(1,46), \mathrm{p}, \eta_{p}^{2}$} & $0.28,0.597,0.006$ & $4.84,0.033,0.095$ & Interaction : & $4,0.714,0.003$ \\
\hline & \multicolumn{4}{|l|}{ Control } \\
\hline $\mathrm{M}(\mathrm{SD})$ & $22.63(5.42)$ & 25.17 & $23.58(6.89)$ & $21.67(3.27)$ \\
\hline $\mathrm{M}(\mathrm{SD})$ & $26.63(4.36)$ & 24.08 & $26.75(4.14)$ & $26.50(4.66)$ \\
\hline \multirow[t]{2}{*}{$\mathrm{F}, \mathrm{p}, \eta_{p}^{2}$} & $22.78,<0.001,0.33$ & $0.89,0.350,0.019$ & Interaction : & $9,0.325,0.021$ \\
\hline & \multicolumn{4}{|c|}{ Real World Disassociation } \\
\hline $\mathrm{M}(\mathrm{SD})$ & $25.58(8.14)$ & 32.10 & $28.67(9.54)$ & $22.50(4.94)$ \\
\hline $\mathrm{M}(\mathrm{SD})$ & $35.77(7.14)$ & 29.25 & $35.54(8.17)$ & $36.00(6.12)$ \\
\hline \multirow[t]{2}{*}{$\mathrm{F}, \mathrm{p}, \eta_{p}^{2}$} & $69.09,<0.001,0.60$ & $2.65,0.110,0.055$ & Interaction : 7 & $31,0.010,0.14$ \\
\hline & \multicolumn{4}{|c|}{ Emotional Involvement } \\
\hline $\mathrm{M}(\mathrm{SD})$ & $24.19(6.45)$ & 26.04 & $24.71(6.66)$ & $23.67(6.34)$ \\
\hline $\mathrm{M}(\mathrm{SD})$ & $26.96(6.59)$ & 25.10 & $27.38(7.24)$ & $26.54(5.99)$ \\
\hline \multirow[t]{2}{*}{$\mathrm{F}, \mathrm{p}, \eta_{p}^{2}$} & $9.71,0.003,0.17$ & $0.31,0.579,0.007$ & \multicolumn{2}{|c|}{ Interaction : $0.014,0.907,0.0003$} \\
\hline & \multicolumn{4}{|c|}{ Cognitive Involvement } \\
\hline $\mathrm{M}(\mathrm{SD})$ & $45.63(7.15)$ & 46.92 & $45.96(7.14)$ & $45.29(7.30)$ \\
\hline $\mathrm{M}(\mathrm{SD})$ & $47.81(7.22)$ & 46.52 & $47.88(8.17)$ & $47.75(6.30)$ \\
\hline $\mathrm{F}, \mathrm{p}, \eta_{p}^{2}$ & $5.15,0.028,0.10$ & $0.045,0.832,0.001$ & Interaction : & $79,0.780,0.002$ \\
\hline & Total & & & \\
\hline $\mathrm{M}(\mathrm{SD})$ & $135.67(24.37)$ & 149.00 & $141.46(28.50)$ & $129.88(18.21)$ \\
\hline $\mathrm{M}(\mathrm{SD})$ & $155.08(22.89)$ & 141.75 & $156.54(25.27)$ & $153.63(20.68)$ \\
\hline $\mathrm{F}, \mathrm{p}, \eta_{p}^{2}$ & $32.58,<0.001,0.42$ & $1.53,0.223,0.032$ & Interaction : 1 & $2,0.209,0.034$ \\
\hline NASA TLX & Physical Demand & & & \\
\hline $\mathrm{M}(\mathrm{SD})$ & $3.52(3.16)$ & 6.23 & $3.04(2.99)$ & $4.00(3.32)$ \\
\hline $\mathrm{M}(\mathrm{SD})$ & $7.27(5.32)$ & 4.56 & $9.42(5.75)$ & $5.13(3.90)$ \\
\hline $\mathrm{F}, \mathrm{p}, \eta_{p}^{2}$ & $44.60,<0.001,0.49$ & $2.51,0.120,0.052$ & Interaction : 2 & $.85,<0.001,0.32$ \\
\hline & Mental Demand & & & \\
\hline $\mathrm{M}(\mathrm{SD})$ & $9.35(4.74)$ & 11.23 & $10.54(4.43)$ & $8.17(4.83)$ \\
\hline $\mathrm{M}(\mathrm{SD})$ & $10.56(5.11)$ & 8.69 & $11.92(5.56)$ & $9.21(4.31)$ \\
\hline $\mathrm{F}, \mathrm{p}, \eta_{p}^{2}$ & $2.98,0.091,0.061$ & $4.49,0.039,0.089$ & Interaction : & $57,0.813,0.001$ \\
\hline & Temporal Demand & & & \\
\hline $\mathrm{M}(\mathrm{SD})$ & $7.15(5.20)$ & 7.79 & $7.29(5.30)$ & $7.00(5.21)$ \\
\hline $\mathrm{M}(\mathrm{SD})$ & $7.25(4.91)$ & 6.60 & $8.29(5.65)$ & $6.21(3.88)$ \\
\hline $\mathrm{F}, \mathrm{p}, \eta_{p}^{2}$ & $0.021,0.886,0.0005$ & $0.88,0.353,0.019$ & Interaction : & $53,0.222,0.032$ \\
\hline & Performance & & & \\
\hline $\mathrm{M}(\mathrm{SD})$ & $9.46(3.87)$ & 9.73 & $9.17(3.80)$ & $9.75(3.99)$ \\
\hline $\mathrm{M}(\mathrm{SD})$ & $9.73(4.15)$ & 9.46 & $10.29(3.90)$ & $9.17(4.40)$ \\
\hline $\mathrm{F}, \mathrm{p}, \eta_{p}^{2}$ & $0.35,0.558,0.008$ & $0.064,0.801,0.001$ & Interaction : : & $47,0.069,0.070$ \\
\hline & Frustration & & & \\
\hline $\mathrm{M}(\mathrm{SD})$ & $9.44(5.07)$ & 9.23 & $9.04(5.01)$ & $9.83(5.21)$ \\
\hline $\mathrm{M}(\mathrm{SD})$ & $8.48(5.13)$ & 8.69 & $9.42(5.44)$ & $7.54(4.73)$ \\
\hline $\mathrm{F}, \mathrm{p}, \eta_{p}^{2}$ & $1.42,0.240,0.030$ & $0.19,0.663,0.004$ & Interaction : & $44,0.105,0.056$ \\
\hline & Effort & & & \\
\hline $\mathrm{M}(\mathrm{SD})$ & $9.44(4.60)$ & 10.19 & $9.92(4.73)$ & $8.96(4.53)$ \\
\hline $\mathrm{M}(\mathrm{SD})$ & $9.42(4.27)$ & 8.67 & $10.46(4.324)$ & $8.38(4.04)$ \\
\hline $\mathrm{F}, \mathrm{p}, \eta_{p}^{2}$ & $0.001,0.976,0.00002$ & $2.04,0.160,0.042$ & Interaction : & $55,0.424,0.014$ \\
\hline
\end{tabular}

Table 1: Statistics for SAM, IEQ, and TLX Results (Sub Scales and Totals as Appropriate) 
that participants experienced less dominance in the laptop condition when they completed the VR condition beforehand $(\mathrm{M}=4.96, \mathrm{SD}=1.08)$, than when the laptop condition was presented first $(\mathrm{M}=5.71, \mathrm{SD}=1.52)$. No significant difference in valence was reported $\mathrm{F}(1,46)=$ $3.57, \mathrm{p}=0.065$. Results support hypothesis H2 (Participants level of arousal will be higher while playing FCD in the VR condition). Figure 5 shows a visualisation of the descriptive statistics for the (within-subject) Laptop and VR conditions.

Anecdotally, higher levels of arousal were also evident in participants' behaviour: we noted that a number of participants verbalised expressions of approval or disappointment in the VR condition, which were less evident when using the laptop.

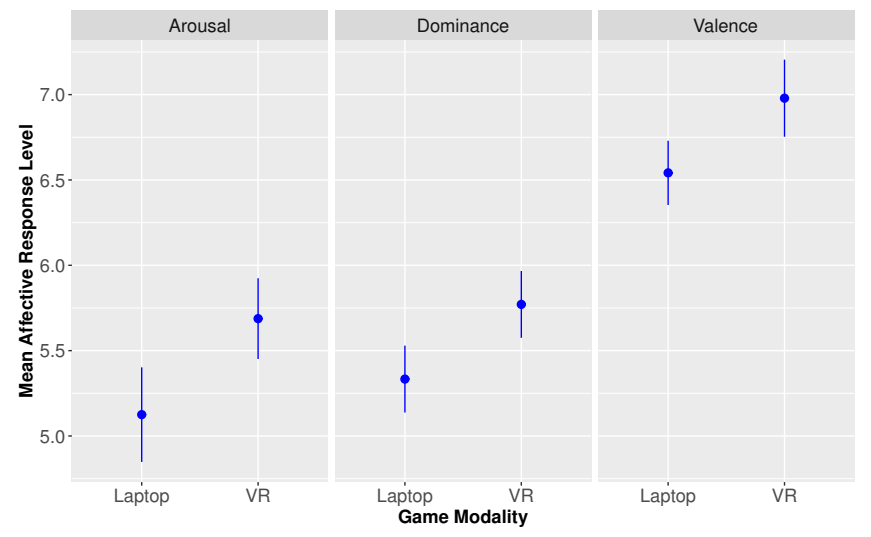

Figure 5: Mean SAM values in Laptop and VR Conditions

\subsection{Task Workloading}

Participants perception of task workload, were recorded using the NASA-TLX self report questionnaire. We processed results in their raw (un-weighted) format, and treated each sub-scale independently (see [59]). The descriptive statistics for the (within-subject) Laptop and VR conditions are visualised in Figure 6.

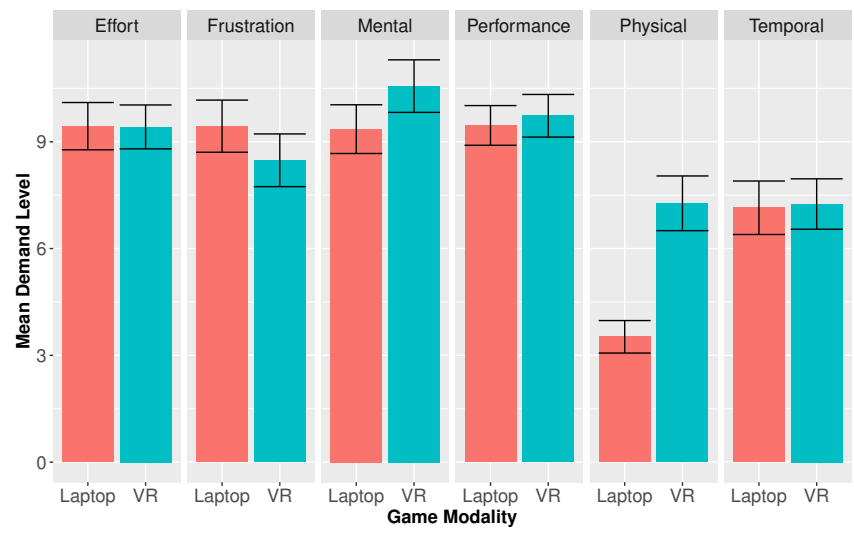

Figure 6: Reported Task Workload Scales, by Condition
No statistically significant main effects were observed with respect to mental demand $(\mathrm{F}(1,46)=2.98, \mathrm{p}=$ $0.091)$, temporal demand $(\mathrm{F}(1,46)=0.021, \mathrm{p}=0.886)$, perceived performance $(\mathrm{F}(1,46)=0.35, \mathrm{p}=0.558)$, effort $(\mathrm{F}(1,46)=0.001, \mathrm{p}=0.976)$ or frustration $(\mathrm{F}(1,46)=1.42$ $, \mathrm{p}=0.24)$. However, participants reported a higher physical demand in the $\mathrm{VR}$ condition $(\mathrm{M}=7.27, \mathrm{SD}=5.32)$ compared to the Laptop condition $(\mathrm{M}=3.52, \mathrm{SD}=3.16)$, and this main effect was statistically significant: $F(1,46)$ $=44.6, \mathrm{p}<0.001, \eta_{p}^{2}=0.49$. Given the large mean difference (3.75) between the VR and laptop conditions, it was not surprising to observe a significant interaction effect between condition type and the order of conditions $\mathrm{F}(1,46)$ $=21.85, \mathrm{p}<0.001, \eta_{p}^{2}=0.32$. The mean levels of physical demand were higher in the VR condition, regardless of the order of presentation; however, participants reported higher mean levels when they completed VR after the laptop condition $(\mathrm{M}=9.42, \mathrm{SD}=5.75)$, in comparison to completing it first $(\mathrm{M}=5.13, \mathrm{SD}=3.9)$. Our results thus only support our hypothesis H3 with respect to physical demand.

\section{Discussion}

This paper explores the potential for the future use of VR as a tool to support gambling behaviour research. We present our study, with 48 participants, that compares game immersion (IEQ), affective response (SAM) and perceived task workload (NASA TLX) while playing the same gambling game in laboratory and VR environments. Results show that participants experienced increased levels of immersion (all sub-scales apart from challenge), arousal, dominance and physical demand in the VR condition. In this section, we discuss the implications of our results for the design of virtual environments, and experimental conditions, when using VR as a platform for future gambling research.

\subsection{System Immersivity, Interface, and Arousal}

Key differences between the VR and laptop versions explored here relate to the user interface: the VR simulation replicates the mechanics of the touchscreen, but arguably exhibits lower system immersivity, as the touch screen has no physical manifestation. However, users also report higher levels of arousal in VR, and this contrasts somewhat with previous works which have typically associated higher system immersivity with higher arousal [34].

However, direct comparisons are complicated by our study design, which uses VR as an additional layer to mediate the experience of playing FCD, and little work has compared experiences in VR with real-world environments. We consider the previous work by Villani et al. 36 to be related to ours: they compared a real experience (of a staged job interview), with a VR simulation in a corporate office, and found higher levels of presence and anxiety in VR. We suggest that our results broadly coincide with 
these findings: while some aspects of the VR betting shop act to undermine users' sense of presence (e.g. not being able to physically touch the screen), the simulation may also provide a more meaningful context for the task, which facilitates higher levels of arousal. This has wider implications; for example, researchers in other sub-fields of psychology, investigating affective responses in different contexts, may benefit from using VR rather laboratorybased experimental conditions.

We also note that other limitations associated with the lack of physicality of VR may serve to undermine the naturalness of participants' experiences in some cases: for example, participants are unable to touch the EGM cabinet, as they would do while playing in real-life. We therefore suggest that researchers may consider using in vivo observational studies to help design effective VR simulations, and to anticipate reduced affordances which might negatively affect participants' experiences. This point also further invites future comparison of VR environments with higher fidelity laboratory conditions (for example, placing a real EGM in a laboratory).

\subsection{Environment and Plausibility}

In general, playing in the VR environment was perceived as more immersive than in the laboratory condition. However, the visual realism of the environment might also increase expectations for other aspects of the simulation to be similarly realistic. This raises an important consideration in the design of VR environments for experimental research: one of the motivations for using VR is to create a more natural and plausible environment to improve the ecological validity of research studies. However, by doing so, (for example, by adding animated characters), the complexity of the experimental environment is increased, and confounds become more difficult to control. In our case, previous work has shown that the presence of observers can affect gambling behaviour in real-world conditions (e.g. 62, 63, 64]) and we therefore did not include computer-controlled characters. Hence, the use of VR as a platform for experimental research re-introduces some of the challenges typically associated with in-the-wild research approaches; researchers designing such VR systems therefore need to be aware of possible confounds, and carefully weigh risks and benefits in the setup of VR research environments.

\subsection{Measuring Experience Within a Virtual Environment}

In our work, we faced the challenge of evaluating the experience of playing the FCD game within VR. While we ensured that we presented the FCD game and VR environment as separate entities to participants, there is potential for ambiguity in the way in which they interpreted their experiences, and also to which components of the conditions (e.g. the VR environment, or the FCD game within VR) certain elements of their experiences should be attributed to. This particularly relates to the IEQ subscales real-world disassociation and control, which tend to focus on the game environment rather than participants' experience of play. For example, when responding to the IEQ item "To what extent did you enjoy the graphics and the imagery?", participants might take this to include the VR environment in the VR condition, but only the FCD game graphics in the Laptop condition. To our knowledge, no existing work has previously evaluated the experience of playing a $2 \mathrm{D}$ digital game while inside a VR simulation; however, this is a potential future use of VR (for example to investigate the affect of environment on video game play), and the investigation of adapted instruments may therefore be warranted.

Our choice of self-reported measures is also a point of reflection. Whilst self-report is well-established, and commonly used to assess experience in both gambling and VR studies, we have just noted that users' experience of gambling in VR is potentially complex. We therefore consider that there is potential for further insights to be gained from the inclusion of more diverse instruments in future studies. We have noted in Section 4.2 that physiological measures such as skin conductance are prone to excessive noise due to movement, and so less suitable for simulated environments (like ours) which support full body motion. However, some recent work 65 has investigated the relationship between self-report and physiological measures in VR studies, and we therefore advocate for the future exploration of physiological measures in full-body motion VR, combined with self-report, to help develop a deeper understanding of user experiences of gambling studies. Furthermore, Skarbez et al. 20] have suggested that behavioural measures, though context specific, may also be useful indicators of experience in VR, and we therefore further suggest that future work could also develop complementary methods of observational analyses.

\subsection{Implications for Gambling Research}

Our study and discussion reveal a number of significant implications for the use of VR as a platform for gambling research. Arousal is known to be an important factor in gambling behaviour (e.g. [28, 53]). Comparisons between real and laboratory environments are sparse, but generally indicate that higher levels of arousal are evident in real conditions $([8,55])$. The reported elevated levels of arousal, dominance and emotional engagement in our study therefore suggest that VR simulations may be better able to evoke emotional experiences which more closely resemble those seen in vivo, as well as creating higher levels of immersion (involvement and focus) while playing. While there may still be some "novelty effect" associated with VR technology, we note that most (39 out of 48) participants had used VR previously.

Participants experienced higher levels of immersion in the FCD game while in the VR simulation. This is a significant finding, as researchers using VR can expect that participants will be more cognitively and emotionally involved in game play, as compared to a laboratory environment. As mentioned in Section 2.3, immersion is characteristic 
of gambling, and so better fostered in VR than in the laboratory. We also note that participants did not report a significant difference in the challenge sub-scale of the IEQ. Whilst this does not imply there is no effect of challenge, we suggest that future work could investigate this aspect of player experience more closely.

These findings support the generalisation of results obtained in VR simulations, and so contribute a number of implications for future gambling behaviour research. Studies in real gambling venues (e.g. [55]), are usually impractical and hard to control, while attempts to recreate more natural conditions in the laboratory (e.g. 8]) also involve significant compromises and limitations. VR allows for the easy creation of simulations with high ecological validity, so that, for example, researchers may design experiments where participants play in different locations in different conditions (for example a casino, a betting shop), or locations which are impractical to access in real life (such as a horse race), with the ability to manipulate and reproduce conditions precisely.

High levels of experimental control also have other methodological implications: for example, study methods in realworld locations are hampered by confounds such as different win/lose outcomes, and changing environmental factors, all of which can be made invariant in simulations. We also point again to previous investigations of the effects of observers on gambling behaviour (62, 63, 64]). We avoided the use of simulated characters in our study, and highlighted the potential pitfalls of doing so; however, there is also opportunity here. It has been shown that participants can respond to VR avatars in similar ways to real people (e.g. 66]), and so with careful study design, VR could be used to replicate more complex human interactions, and explore the influence of those interactions on gamblers' behaviour with a level of control which is impractical in vivo.

\subsection{Implications for the Simulation of Other Physical Sys- tems}

While our work is contextualised by gambling research, our findings also have implications for other areas of research, and for designers of other types of physical systems. For example, a separate and significant body of research addresses the experience of players of (non-gambling) video games, and similarly considers the validity of laboratory based experimental work (e.g. 67]). Our results suggest that such work could also benefit from the use of VR simulations of natural play environments to create greater ecological validity, and so foster player immersion and more representative player experiences; for example, by recreating arcade or home environments.

Our work could further benefit both research and training applications related to other types of interactive systems. VR is already used extensively for training (see Jensen et al. 68 for a recent review), and sometimes used to simulate conditions which are hard to reproduce in real life. However, we suggest that our results support the use of VR simulations for training users of electronic or computerised equipment in specialist environments (for example, industrial settings), which may better reproduce levels of user immersion in operational tasks than nonnatural training settings. As a limitation, we refer back to our previous points regarding lack of physicality, as this is also a consideration for applications outside of gambling research.

\section{Limitations and Future Work}

We have already highlighted some limitations of our study: our VR environment is detailed, but lacks some features which might add to players' sense of presence, and these warrant exploration. In particular, the inclusion of other characters (whether autonomous or otherwise) may increase players' feelings of plausibility. We have discussed the potential advantages and problems of including characters in experimental conditions in Section 6, but we suggest that this is an interesting direction for further study.

A much less easily addressed limitation relates to the use of in-game (rather than real) currency: this limitation applies to most laboratory work, and is also subject to ethical considerations, but ultimately limits the ecological validity of much gambling behaviour research. This also relates to a wider limitation of our study: we propose that VR environments are more representative of real-world conditions than the laboratory, but we have not compared directly with in vivo conditions. We therefore propose that further work might engage with this, to help further establish the effectiveness of VR.

We have conducted our experiments with a sample of 48 participants, and detected a number of statistically significant effects. However, we acknowledge some limitations: a number of sub-scales (for example, on the TLX, and the valence scale on the SAM) indicated small effect sizes, and so it is possible that there are a number of additional effects which could be detected by replicating with larger sample sizes. However, we also suggest that future work could focus on some specific aspects of user experience (such as usability of the VR equipment) in more detail, or using different measures (such as behavioural), in order to mitigate this limitation. In addition, we note the evidence of interactions between condition order and condition type in three of the fifteen sub-scales. While these do not significantly affect our main findings, we suggest that the possibility of contrast effects in some aspects of user experience (particularly real-world disassociation) are worthy of further consideration, and may have implications for the design of future studies.

Finally, we note that for our study we did not select participants based on gambling experience. While we assert the generality of our results, many gambling studies do specifically employ regular or problem gamblers as participants. We therefore suggest that future work could further investigate the use of VR with these specific groups, and 
might help to develop more valid and naturalistic simulations.

\section{Conclusions}

We have compared the experiences of participants playing a representative touchscreen gambling game under two conditions: one on a laptop in a laboratory environment, and the other on a virtual gambling machine, in a virtual reality representation of a betting shop. No previous work has made a comparable investigation of perceived immersion or affective response in this context.

We hypothesised that participants would experience higher levels of arousal and immersion in the game while in the VR condition. Our study $(\mathrm{N}=48)$ confirmed our hypotheses using self-report measures, although no significant difference in challenge while playing was reported. Participants also reported higher perceived physical workloading in VR, probably reflecting some level of encumbrance while using the VR interface.

Based on our results, we argue for the use of VR in future research into gambling behaviour over laboratory based studies. VR environments not only promote higher levels of engagement with the experimental task, but also promote increased levels of immersion and emotional response which we suggest are more representative of realworld responses. However, we also argue for further work to extend and confirm our findings, particularly regarding the use of virtual characters to create a greater sense of presence, and also to directly compare our results with those obtained in vivo, using experienced gamblers.

\section{References}

[1] NatCen Social Research (for the UK Gambling Commission), Gambling behaviour in great britain in 2015 (August 2017).

[2] UK Gambling Commission, Industry statistics, april 2014 to march 2017 (updated to include october 2016 to september 2017) (May 2018).

[3] S. Monaghan, A. Blaszczynski, Impact of mode of display and message content of responsible gambling signs for electronic gaming machines on regular gamblers, Journal of Gambling Studies 26 (1) (2010) 67-88. doi:10.1007/s10899-009-9150-z

[4] J. Parke, M. Griffiths, The psychology of the fruit machine: The role of structural characteristics (revisited), International Journal of Mental Health and Addiction 4 (2) (2006) 151-179. doi:10.1007/s11469-006-9014-z.

[5] L. Dixon, R. Trigg, M. Griffiths, An empirical investigation of music and gambling behaviour, International Gambling Studies 7 (3) (2007) 315-326. doi:10.1080/14459790701601471

[6] S. M. Gainsbury, L. Jakob, D. Aro, Understanding enduser perspectives to enhance perceived value uptake of harmminimization tools: considering gamblers views of a precommitment system, International Gambling Studies 18 (1) (2018) 22-38. doi:10.1080/14459795.2017.1370723

[7] A. Harris, M. D. Griffiths, The impact of speed of play in gambling on psychological and behavioural factors: A critical review, Journal of Gambling Studies 34 (2) (2018) 393-412. doi:10.1007/s10899-017-9701-7.

[8] G. Anderson, R. I. F. Brown, Real and laboratory gambling, sensation-seeking and arousal, British Journal of Psychology 75 (3) 401-410. doi:10.1111/j.2044-8295.1984.tb01910.x
[9] S. Gainsbury, A. Blaszczynski, The appropriateness of using laboratories and student participants in gambling research, Journal of Gambling Studies 27 (1) (2011) 83-97. doi:10.1007/ s10899-010-9190-4

[10] S. Bouchard, S. Dumoulin, G. Robillard, T. Guitard, . Klinger, H. Forget, C. Loranger, F. X. Roucaut, Virtual reality compared with in vivo exposure in the treatment of social anxiety disorder: A three-arm randomised controlled trial, British Journal of Psychiatry 210 (4) (2017) 276283. doi:10.1192/bjp.bp.116. 184234

[11] C. Botella, B. Serrano, R. Baos, A. Garcia-Palacios, Virtual reality exposure-based therapy for the treatment of posttraumatic stress disorder: a review of its efficacy, the adequacy of the treatment protocol, and its acceptability., Neuropsychiatric disease and treatment 11 (2015) 2533-2545. doi:doi: 10.2147/NDT.S89542

[12] C. Botella, J. Fernández-Álvarez, V. Guillén, A. GarcíaPalacios, R. Baños, Recent progress in virtual reality exposure therapy for phobias: A systematic review, Current Psychiatry Reports 19 (7). doi:10.1007/s11920-017-0788-4.

[13] P. Lindner, A. Miloff, S. Fagerns, J. Andersen, M. Sigeman, G. Andersson, T. Furmark, P. Carlbring, Therapist-led and selfled one-session virtual reality exposure therapy for public speaking anxiety with consumer hardware and software: A randomized controlled trial, Journal of Anxiety Disorders 61 (2019) 45 - 54. doi:https://doi.org/10.1016/j.janxdis.2018.07.003.

[14] C.-B. Park, S. M. Park, A. R. Gwak, B. K. Sohn, J.-Y. Lee, H. Y. Jung, S.-W. Choi, D. J. Kim, J.-S. Choi, The effect of repeated exposure to virtual gambling cues on the urge to gamble, Addictive Behaviors 41 (2015) $61-64$. doi:10.1016/j. addbeh.2014.09.027

[15] S. Bouchard, G. Robillard, I. Giroux, C. Jacques, C. Loranger, M. St-Pierre, M. Chrtien, A. Goulet, Using virtual reality in the treatment of gambling disorder: The development of a new tool for cognitive behavior therapy, Frontiers in Psychiatry 8 (2017) 27. doi:10.3389/fpsyt.2017.00027

[16] J. Steuer, Defining virtual reality: Dimensions determining telepresence, Journal of Communication 42 (4) (1992) 73-93. doi:10.1111/j.1460-2466.1992.tb00812.x

[17] B. G. Witmer, M. J. Singer, Measuring presence in virtual environments: A presence questionnaire, Presence: Teleoperators and Virtual Environments 7 (3) (1998) 225-240. doi: 10.1162/105474698565686

[18] M. Slater, S. Wilbur, A framework for immersive virtual environments (five): Speculations on the role of presence in virtual environments, Presence: Teleoperators and Virtual Environments 6 (6) (1997) 603-616. doi:10.1162/pres.1997.6.6.603

[19] M. Slater, Place illusion and plausibility can lead to realistic behaviour in immersive virtual environments, Philosophical Transactions of the Royal Society of London B: Biological Sciences 364 (1535) (2009) 3549-3557. doi:10.1098/rstb.2009.0138.

[20] R. Skarbez, F. P. Brooks, Jr., M. C. Whitton, A survey of presence and related concepts, ACM Comput. Surv. 50 (6) (2017) 96:1-96:39. doi:10.1145/3134301.

[21] T. Schubert, F. Friedmann, H. Regenbrecht, The experience of presence: Factor analytic insights, Presence: Teleoperators and Virtual Environments 10 (3) (2001) 266-281. doi:10.1162/ 105474601300343603

[22] M. Slater, A note on presence terminology, Presence Connect 3 (2003) 1-5.

[23] E. Brown, P. Cairns, A grounded investigation of game immersion, in: CHI '04 Extended Abstracts on Human Factors in Computing Systems, CHI EA '04, ACM, New York, NY, USA, 2004, pp. 1297-1300.

[24] C. Jennett, A. L. Cox, P. Cairns, S. Dhoparee, A. Epps, T. Tijs, A. Walton, Measuring and defining the experience of immersion in games, International Journal of Human-Computer Studies 66 (9) (2008) $641-661$. doi:https://doi.org/10.1016/j. ijhcs.2008.04.004

[25] M. Csikszentmihalyi, Flow: The Psychology of Optimal Experience, Harper Perennial, New York, NY, USA, 1990. 
[26] P. Sweetser, P. Wyeth, Gameflow: A model for evaluating player enjoyment in games, Comput. Entertain. 3 (3) (2005) 3-3. doi : 10.1145/1077246.1077253

[27] M. Slater, Measuring presence: A response to the witmer and singer presence questionnaire, Presence: Teleoperators and Virtual Environments 8 (5) (1999) 560-565. doi:10.1162/ 105474699566477 .

[28] Levels of arousal in high- and low-frequency gamblers, Behaviour Research and Therapy 23 (6) (1985) 635 - 640. doi: https://doi.org/10.1016/0005-7967(85)90058-0

[29] E. Wulfert, B. D. Roland, J. Hartley, N. Wang, C. Franco, Heart rate arousal and excitement in gambling: Winners versus losers., Psychology of Addictive Behaviors 19 (2005) 311-316. doi: http://dx.doi.org/10.1037/0893-164X.19.3.311

[30] M. Dickerson, S. Adcock, Mood, arousal and cognitions in persistent gambling: Preliminary investigation of a theoretical model, Journal of gambling behavior 3 (1) (1987) 3-15. doi:10.1007/BF01087473

31] G. Riva, F. Mantovani, C. S. Capideville, A. Preziosa, F. Morganti, D. Villani, A. Gaggioli, C. Botella, M. Alcaiz, Affective interactions using virtual reality: The link between presence and emotions, CyberPsychology \& Behavior 10 (1) (2007) 4556. doi:10.1089/cpb.2006.9993

[32] S. Estupiñán, F. Rebelo, P. Noriega, C. Ferreira, E. Duarte, Can virtual reality increase emotional responses (arousal and valence)? a pilot study, in: A. Marcus (Ed.), Design, User Experience, and Usability. User Experience Design for Diverse Interaction Platforms and Environments, Springer International Publishing, 2014, pp. 541-549.

[33] K. Kim, M. Z. Rosenthal, D. J. Zielinski, R. Brady, Effects of virtual environment platforms on emotional responses, Computer Methods and Programs in Biomedicine 113 (3) (2014) 882 - 893. doi:https://doi.org/10.1016/j.cmpb.2013.12.024

[34] J. Diemer, G. W. Alpers, H. M. Peperkorn, Y. Shiban, A. Mhlberger, The impact of perception and presence on emotional reactions: a review of research in virtual reality, Frontiers in Psychology 6 (2015) 26. doi:10.3389/fpsyg.2015.00026

[35] V. T. Visch, E. S. Tan, D. Molenaar, The emotional and cognitive effect of immersion in film viewing, Cognition and Emotion 24 (8) (2010) 1439-1445. doi:10.1080/02699930903498186

[36] D. Villani, C. Repetto, P. Cipresso, G. Riva, May i experience more presence in doing the same thing in virtual reality than in reality? an answer from a simulated job interview, Interacting with Computers 24 (4) (2012) 265-272. doi:10.1016/j.intcom. 2012.04.008

[37] A. Garcia-Palacios, H. Hoffman, A. Carlin, T. Furness, C. Botella, Virtual reality in the treatment of spider phobia: a controlled study, Behaviour Research and Therapy 40 (9) (2002) 983 - 993. doi:10.1016/S0005-7967(01)00068-7

[38] M. Krijn, P. Emmelkamp, R. Biemond, C. De Wilde De Ligny, M. Schuemie, C. Van Der Mast, Treatment of acrophobia in virtual reality: The role of immersion and presence, Behaviour Research and Therapy 42 (2) (2004) 229-239. doi:10.1016/ S0005-7967 (03)00139-6

[39] M. Krijn, P. Emmelkamp, R. lafsson, M. Bouwman, L. Van Gerwen, P. Spinhoven, M. Schuemie, C. Van Der Mast, Fear of flying treatment methods: Virtual reality exposure vs. cognitive behavioral therapy, Aviation Space and Environmental Medicine 78 (2) (2007) 121-128.

[40] L. R. Valmaggia, L. Latif, M. J. Kempton, M. Rus-Calafell, Virtual reality in the psychological treatment for mental health problems: An systematic review of recent evidence, Psychiatry Research 236 (2016) 189 - 195. doi:https://doi.org/10.1016/ j.psychres.2016.01.015

[41] A. Gaggioli, Using virtual reality in experimental psychology, in: G. Riva, C. Galimberti (Eds.), Towards cyberpsychology: Mind, cognition and society in the internet age, IOP Press, Amsterdam, 2001, Ch. 9, pp. 157-174.

42] C. Wilson, A. Soranzo, The use of virtual reality in psychology: A case study in visual perception, Computational and Mathematical Methods in Medicine 2015, Art. ID 151702. doi:10.1155/2015/151702

[43] C. J. Bohil, B. Alicea, F. A. Biocca, Virtual reality in neuroscience research and therapy, Nature Reviews Neuroscience 12 (12) (2011) 752-762.

[44] X. Pan, A. F. d. C. Hamilton, Why and how to use virtual reality to study human social interaction: The challenges of exploring a new research landscape, British Journal of Psychology 109 (3) (2018) 395-417.

[45] A. Kulik, Virtually the ultimate research lab, British Journal of Psychology 109 (3) (2018) 434-436.

[46] I. Giroux, A. Faucher-Gravel, A. St-Hilaire, C. Boudreault, C. Jacques, S. Bouchard, Gambling exposure in virtual reality and modification of urge to gamble, Cyberpsychology, Behavior, and Social Networking 16 (3) (2013) 224-231. doi: 10.1089/cyber. 2012.1573

[47] C. Loranger, S. Bouchard, J. Boulanger, G. Robillard, Validation of two virtual environments for the prevention and treatment of pathological gambling.(addictions, urges and cravings: Towards better understanding and treatments)(report), Journal of CyberTherapy and Rehabilitation 4 (2) (2011) 233-235.

[48] M. Young, M. Wohl, K. Matheson, S. Baumann, H. Anisman, The desire to gamble: The influence of outcomes on the priming effects of a gambling episode, Journal of Gambling Studies 24 (3) (2008) 275-293. doi:10.1007/s10899-008-9093-9

[49] M. J. Wohl, A. Parush, H. A. S. Kim, K. Warren, Building it better: Applying humancomputer interaction and persuasive system design principles to a monetary limit tool improves responsible gambling, Computers in Human Behavior 37 (2014) 124 - 132. doi:https://doi.org/10.1016/j.chb.2014.04.045.

[50] J. J. Lister, L. Nower, M. J. Wohl, Gambling goals predict chasing behavior during slot machine play, Addictive Behaviors 62 (2016) 129 - 134. doi : https://doi.org/10.1016/j.addbeh. 2016.06 .018

[51] S. J. Hollingshead, M. J. Wohl, D. Santesso, Do you read me? including personalized behavioral feedback in pop-up messages does not enhance limit adherence among gamblers, Computers in Human Behavior 94 (2019) 122 - 130. doi :https://doi.org/ 10.1016/j.chb.2019.01.015.

[52] H. J. Pijeira-Daz, H. Drachsler, S. Jrvel, P. A. Kirschner, Sympathetic arousal commonalities and arousal contagion during collaborative learning: How attuned are triad members?, Computers in Human Behavior 92 (2019) 188 - 197. doi:https: //doi.org/10.1016/j.chb.2018.11.008

[53] M. Griffiths, Tolerance in gambling: An objective measure using the psychophysiological analysis of male fruit machine gamblers, Addictive Behaviors 18 (3) (1993) 365 - 372. doi:https://doi. org/10.1016/0306-4603(93) 90038-B

[54] K. R. Coventry, J. Hudson, Gender differences, physiological arousal and the role of winning in fruit machine gamblers, Addiction 96 (6) (2001) 871-879. doi:10.1046/j.1360-0443. 2001.9668718.x

[55] K. M. Diskin, D. C. Hodgins, S. A. Skitch, Psychophysiological and subjective responses of a community sample of video lottery gamblers in gambling venues and laboratory situations, International Gambling Studies 3 (2) (2003) 133-148. doi:10.1080/1356347032000142243

[56] N. D. Schull, Digital gambling: The coincidence of desire and design, The ANNALS of the American Academy of Political and Social Science 597 (1) (2005) 65-81. doi:10.1177/ 0002716204270435

[57] M. J. Dixon, M. Stange, C. J. Larche, C. Graydon, J. A. Fugelsang, K. A. Harrigan, Dark flow, depression and multiline slot machine play, Journal of Gambling Studies 34 (1) (2018) 73-84. doi:10.1007/s10899-017-9695-1

[58] W. S. Murch, L. Clark, Effects of bet size and multi-line play on immersion and respiratory sinus arrhythmia during electronic gaming machine use, Addictive Behaviors 88 (2019) $67-72$. doi:https://doi.org/10.1016/j.addbeh.2018.08.014

[59] S. G. Hart, Nasa-task load index (nasa-tlx); 20 years later, Proceedings of the Human Factors and Ergonomics Society Annual Meeting 50 (9) (2006) 904-908. doi:10.1177/ 


\section{9}

[60] Measuring emotion: The self-assessment manikin and the semantic differential, Journal of Behavior Therapy and Experimental Psychiatry 25 (1) (1994) 49 - 59. doi:https://doi. org/10.1016/0005-7916(94)90063-9

[61] T. D. Parsons, Virtual reality for enhanced ecological validity and experimental control in the clinical, affective and social neurosciences, Frontiers in Human Neuroscience 9 (2015) 660

[62] H. Molde, R. Mentzoni, D. Hanss, D. Sagoe, S. L. Andersen, S. Pallesen, People around you do they matter? an experimental gambling study, International Gambling Studies 17 (3) (2017) 349-365. doi:10.1080/14459795.2017.1333130

[63] S. Mishra, M. Morgan, M. L. Lalumière, R. J. Williams, Mood and audience effects on video lottery terminal gambling, Journal of Gambling Studies 26 (3) (2010) 373-386. doi:10.1007/ s10899-009-9158-4

[64] J. E. Lemoine, C. Roland-Lvy, The effect of the presence of an audience on risk-taking while gambling: the social shield, Social Influence 12 (2-3) (2017) 101-114. doi:10.1080/15534510. 2017.1373697.

[65] D. Egan, S. Brennan, J. Barrett, Y. Qiao, C. Timmerer, N. Murray, An evaluation of heart rate and electrodermal activity as an objective qoe evaluation method for immersive virtual reality environments, in: 2016 Eighth International Conference on Quality of Multimedia Experience (QoMEX), 2016, pp. 1-6. doi:10.1109/QoMEX.2016.7498964

[66] K. Zibrek, E. Kokkinara, R. McDonnell, Don't stand so close to me: Investigating the effect of control on the appeal of virtual humans using immersion and a proximity-based behavioral task, in: Proceedings of the ACM Symposium on Applied Perception, SAP '17, ACM, New York, NY, USA, 2017. doi:10.1145/3119881.3119887

[67] J. Takatalo, J. Hkkinen, J. Kaistinen, G. Nyman, User experience in digital games: Differences between laboratory and home, Simulation \& Gaming 42 (5) (2011) 656-673. doi: 10.1177/1046878110378353

[68] L. Jensen, F. Konradsen, A review of the use of virtual reality head-mounted displays in education and training, Education and Information Technologies 23 (4) (2018) 1515-1529. doi: $10.1007 / \mathrm{s} 10639-017-9676-0$ 